\title{
Basaloid Squamous Cell Carcinoma and Adenoid Cystic Carcinoma of Uterine Cervix: A Report of Three Cases With Comparison of Clinicopathological, Immunohistochemical, and Ultrastructural Findings
}

\section{Soon Auck Hong}

Chung-Ang University College of Medicine

Su Hyun Yoo ( $\sim$ S5596@paik.ac.kr)

Inje University Sanggye Paik Hospital

\section{Case Report}

Keywords: Basaloid squamous cell carcinoma, adenoid cystic carcinoma, cervix

Posted Date: February 23rd, 2022

DOI: https://doi.org/10.21203/rs.3.rs-1374880/v1

License: (c) (1) This work is licensed under a Creative Commons Attribution 4.0 International License. Read Full License 


\section{Abstract}

Background: Basaloid squamous cell carcinoma (BSCC) is a rare, aggressive variant of squamous cell carcinoma with high stage presentations and poor outcomes. It is especially difficult to differentiate BSCC with an adenoid cystic pattern from high grade adenoid cystic carcinoma (ACC) using small biopsies.

Case presentation: We describe different clinicopathological, immunohistochemical, and ultrastructural findings of BSCC $(n=2)$ and ACC $(n=1)$ of the uterine cervix. Despite similar histopathologic features, BSCC had significantly higher cellular atypism and distinct squamous differentiation, and showed diffuse immunoreactivity to P16 and P63, but non-specific pattern for S100, p53, and CD117 compared with ACC, which showed selective peripheral/internalized positivity for P63 and S100 in myoepithelial cells, and diffuse expression of P53 and P16. High risk Human Papilloma Virus (HPV) types (types 16 and 56) were identified in BSCC, but not in ACC. However, BSCC had relatively undifferentiated cellular characteristics and ACC showed occasional true ductal differentiation with luminal microvilli on ultrastructural studies.

Conclusions: $\mathrm{p} 16$ overexpression is not a reliable surrogate marker of HPV in relation to differentiated basaloid SCC from high-grade ACC. Useful tools to distinguish a 'basaloid carcinoma of the cervix' should be accompanied by HPV analysis and immunostaining patterns for P63 and S100.

\section{Background}

Basaloid squamous cell carcinoma (BSCC) is a more aggressive variant of squamous cell carcinoma with a predilection for the aerodigestive tract, including the head and neck[1], esophagus[2], anal canal[3], lung[4], and a rare prevalence for the urinary bladder[5], cervix, and vulva. Cervical BSCC is a rare entity that is under-recognized. However, adenoid cystic carcinoma (ACC) appears to be less aggressive compared to the usual types of squamous cell carcinoma. Both tumors are recognized as separate entities from the usual type of squamous cell carcinoma due to their different clinical outcomes. Histologically, cervical BSCC has a similarity to ACC and is characterized by eosinophilic basement membrane-like material and cribriform tumor lobules. It can be especially difficult to differentiate BSCC from adenoid cystic characteristics from high-grade ACC in small biopsies. In this paper, we describe three cases, including 2 cases of BSCC and one case of ACC, of the uterine cervix in old women. We investigated whether immunohistochemical staining of p63, p53, p16, CD117, smooth muscle actin (SMA) and S100 protein, periodic acid-Schiff stain, HPV real-time PCR, and electron microscopic examination could be useful tools of distinguishing the 'basaloid carcinoma of the cervix'.

\section{Case Presentation}

\section{Case No. 1}

An 82-year-old woman was transferred to our hospital under the impression of cervical mass after diagnosis of ASC-US on cytology. She was examined for baseline values. She had no significant medical 
history except hypertension and hyperlipidemia. On magnetic resonance imaging, an approximately 5.2 $\mathrm{cm}$ mass in the uterine cervix was noted without evidence of parametrial invasion. The tumor was classified as clinical stage Ib2. A punch biopsy was performed, and a tumor composed of hyperchromatic cells with scanty cytoplasm and frequent mitoses, showing infrequently cystic spaces containing mucinlike material was noted. Differential diagnoses included poorly differentiated adenocarcinoma, poorly differentiated squamous cell carcinoma, high-grade adenoid cystic carcinoma, large cell neuroendocrine carcinoma, endometrial stromal sarcoma, and leiomyosarcoma. Immunohistochemical stains for ER, PR, p53, chromogranin, synaptophysin, S100 protein, and CD10, were negative; however, there was diffuse positivity for p16 and pan-cytokeratin immunostains. The tumor was diagnosed as high-grade carcinoma, which did not determine specific differentiation. She underwent radical hysterectomy with bilateral salpingo-oophorectomy and pelvic lymph node dissection. The resected specimen showed a well-defined $5 \mathrm{~cm}$-sized mass on the cervix. The tumor invaded the parametrial tissues and uterine myometrium beyond the entire cervical wall. There was no metastasis to any pelvic lymph nodes.

Histologically, the tumor was composed of small monotonous cells with scant cytoplasm, partly arranged with palisading appearance, and partly cribriform tumor lobules (Fig. 1A). The eosinophilic thick basement membrane surrounding tumor nests and cribriform tumor lobules was stained a magenta color by PAS stain. Focal distinct squamous differentiation was noted at the periphery of the tumor (Fig. 1D). Additional axillary studies were performed to confirm the diagnosis. The tumor showed diffuse immunoreactivity for p63 (Fig. 1G) and p16 (Fig. 1M), but was immunonegative for S100 protein (Fig. $1 J)$.

Ultrastructural studies demonstrated relatively undifferentiated cells consisting of a cystic lumen without their own luminal microvilli (Fig. 1P). HPV type 16 detected by real-time polymerase chain reaction (PCR) was noted for this case (Table 1). A diagnosis of HPV-associated basaloid squamous cell carcinoma (BSCC) was made on the basis of the histological, immunohistochemical, and ultrastructural findings. 
Table 1

Clinicopathologic findings between basaloid squamous cell carcinoma and adenoid cystic carcinoma

\section{Case 1}

Age (yrs)

82

Abnormal cytology

$\begin{array}{ll}\begin{array}{ll}\text { Chief } \\ \text { complaints }\end{array} & \text { Abnorma } \\ & \text { (ASC-US) }\end{array}$

Tumor size
Clinical stage
Adjuvant
chemotherapy

Follow-up

No evidence of recurrence $(3 \mathrm{mo})$

Lost follow-up

Focal +

Squamous

Focal +

$4 \mathrm{~cm}$

IB2

$3.7 \mathrm{~cm}$

IB3

IB2

Case 3

80

Vaginal bleeding

Vaginal bleeding

$+$

$+$

No evidence of recurrence (14mo)

Pathologic findings

differentiation

Ductal

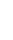

differentiation

Immunohistochemical stainings

p63

$+++$

$+++$

+ (Peripheral/internalized positivity)

p53

S 100 protein

p16

$+++$

$+++$

CD117

Focal +, (membranous)

-
-
+++

SMA

Special stain

Periodic acid- $\quad+$; basement membrane $\quad+$; basement membrane Schiff +; eosinophilic basement membrane-like material

Molecular study

HPV real time HR HPV 16

HR HPV 56

PCR

Electron microscopic examination 


\begin{tabular}{|lll|}
\hline Case 1 & Case 2 & Case 3 \\
\hline $\begin{array}{l}\text { Thick basement } \\
\text { membrane, }\end{array}$ & $\begin{array}{l}\text { Thick basement } \\
\text { membrane, }\end{array}$ & $\begin{array}{l}\text { Ductal differentiation with } \\
\text { luminal microvilli }\end{array}$ \\
$\begin{array}{l}\text { Undifferentiated } \\
\text { cellular characteristics }\end{array}$ & $\begin{array}{l}\text { Undifferentiated } \\
\text { cellular characteristics }\end{array}$ & \\
\hline
\end{tabular}

\section{Case No. 2}

A 74-year-old woman with a symptom of vaginal bleeding underwent physical examinations and surgical resection at an outside hospital. A 4-cm-sized polypoid mass was identified on the uterine cervix. The patient underwent total vaginal hysterectomy. The tumor had invaded into two-thirds of the thickness of the entire cervical wall and had no evidence of extension to the vagina and endomyometrium. There was no evidence of lymphovascular and neural invasion. This tumor was composed of partly solid monotonous round cells and partly epithelial cells with cords or a cribriform pattern (Fig. 1B). The cords or nests of small monotonous cells were arranged in the myxoid stromal change. These tumor components were considered part of the spectrum of the same tumor type without a high-grade sarcomatous component by extensive gross examination.

It was difficult to determine the correct diagnosis because the histologic findings were similar to those of ACC with a solid variant, showing focal squamous differentiation. This tumor displayed eosinophilic basement membrane-like material and cribriform tumor lobules. The periphery of the tumor showed a basaloid cell nest consisting of a palisading arrangement with squamous differentiation (Fig. 1E). Immunohistochemistry demonstrated that the tumor cells were strongly positive for p63 (Fig. $1 \mathrm{H})$ and p16 (Fig. 1N), but not S100 protein (Fig. 1K). The undifferentiated cells had a nucleoli arranged cystic lumen without luminal microvilli as determined by ultrastructural studies (Fig. 1Q). HPV high-risk 56 subtype was detected by real-time PCR in this case (Table 1). She has had no evidence of recurrence during a follow-up period of 36 months.

\section{Case No. 3}

An 80-year-old woman was referred to our hospital from a local hospital for vaginal bleeding. She was examined using a punch biopsy at the local hospital, which was suspicious for adenoid cystic carcinoma. Magnetic resonance imaging showed a 3.7-cm-sized mass, confined to the cervical wall with no evidence of involvement of the vagina. The tumor was classified as clinical stage lb1. The tumor was a biphasic neoplasm comprised of ductal and myoepithelial components. The growth pattern of this tumor showed cribriform, glandular, and solid forms containing basement membrane-like materials (Fig. 1C). At the periphery, ACC showed some glandular structures with true ductal differentiation (Fig. 1F), having luminal microvilli detected by ultrastructural studies (Fig. 1R). ACC displayed selective peripheral/internalized positivity for p63 (Fig. 1I), S100 protein (Fig. 1L), and CD117 in myoepithelial cells, and diffuse expression of p16 (Fig. 10) with no detection of HPV infection (Table 1). On ultrastructural studies, the 
cystic lumen showed lamina and microvilli (Fig. 1R). Such structures are recapitulated in glandular epithelial differentiation. She had undergone radiotherapy for the cervical mass. After radiotherapy, she received simple hysterectomy with bilateral salpingo-oophorectomy. The tumor measured $3.0 \mathrm{~cm}$ in the longest dimension and extended to the pericervical adipocytic tissue and showed endomyometrial extension. During the follow-up period of 14 months, she was free of recurrence.

\section{Discussion}

BSCC of the uterine cervix is a rare subtype as defined by the most recent WHO classification. BSCC is considered an aggressive squamous cell carcinoma variant, which has received little attention in the literature. [6] It is difficult to make a distinct diagnosis of BSCC from biopsy specimens because there are various spectrums of high-grade basaloid tumors in the cervix, particularly ACC, which may be similar. Some papers have reported that BSCC in the anus and esophagus displayed an adenoid cystic pattern,

which resembled ACC of the head and neck. $[3,7]$ The hyalinized stroma around the tumor, eosinophilic basement membrane, and cystic changes can be confused with solid, high-grade ACC. The correct diagnosis from BSCC to ACC is important because of its worse prognosis.

ACC is also a rare tumor arising most commonly in the salivary and lacrimal glands and rarely other organs, such as the skin, breast, lung, uterine cervix, and vulva. [8] ACC in the uterine cervix has been reported to be associated with high-risk HPV infection in some cases.[9] However, data between p16 expression and HPV infection in high-grade ACC is limited.

In case 2, it was especially difficult to distinguish BSCC from ACC with a solid variant. It showed a cribriform pattern of basaloid cell proliferation with variable amounts of myxoid material. Additional sections were obtained from the hysterectomy specimen for further evaluation. The periphery of the tumor showed a basaloid cell nest consisting of a palisading arrangement with distinct squamous differentiation (Fig. 1E). The immunoprofile of the tumor showed diffuse reactive staining for p63 (Fig. 1G) and p16 (Fig. 1M), but was immunonegative for S100 protein (Fig. 1J) unlike ACC. BSCCs displayed diffuse immunoreactivity for p63 whereas ACC showed a compartmentalized pattern within tumor nests, in which p63-positive cells were found internally as well as at the periphery.

Squamous cell carcinoma of the uterine cervix, including basaloid squamous cell carcinoma, is associated with HPV infection. Diffuse strong positivity of p16 immunohistochemical staining is a wellknown surrogate marker for carcinoma associated with HPV infection. The p16 gene (INK4a/CDKN2A) is a tumor suppressor gene that encodes a cyclin-dependent kinase inhibitor involved in cell cycle regulation and which inhibits $\mathrm{Rb}$ phosphorylation leading to cell cycle arrest and a negative feedback loop to control cell proliferation. During high-risk HPV infection, DNA integrates into the genome of the host squamous cells, the viral oncoprotein E7 binds to the retinoblastoma protein, and induces its functional inactivation. The overexpression of p16 appears due to the loss of negative feedback.[10]

Diffuse strong p16 overexpression has been described in ACC of the head and neck; however, the association of HPV infection with ACC is controversial. Boland et al. reported that only $11 \%$ of high-grade 
ACCs of the head and neck were combined with high-risk HPV infection among high grade ACC showing immunopositivity for $\mathrm{p} 16$. These results suggested that the presence of HPV infection in high-grade ACC was not paralleled by the strong expression of p16. [11] Although ACC of the uterine cervix may be accompanied by other HPV-related lesions[12], p16 immunopositivity does not always reflect HPV infection status in ACC. p16 overexpression can result from different mechanisms independent of HPV, such as $C D K N 2 A$ gene mutations, deletions, and promoter hypermethylation.[13] All three cases of 'basaloid carcinoma of the uterine cervix' underwent immunohistochemistry for p16 staining and molecular analysis by HPV real-time PCR in this study. All three cases showed diffuse strong expression of p16. The presence of high-risk HPV was revealed in 2 BSCC cases; however, HPV infection was not detected in the ACC case.

There are distinctive ultrastructural features between BSCC and high-grade ACC by electron microscopy. The polygonal cells of BSCC and ACC had a thick basement membrane and reduplicated external lamina. Also, there was no distinctive differentiation in nuclear morphology. In BSCCs from cases 1 and 2, tumors showed relatively undifferentiated cells consisting of cystic lumen without their luminal microvilli; however, ACC showed occasional ductal differentiation with luminal microvilli.

In conclusion, diffuse strong p16 expression in cervical cancer is generally associated with HPV infection. However, it is not a reliable surrogate marker of HPV in relation to ACC. Therefore, the use of other special methods is important to differentiate basaloid SCC from high-grade ACC. In cervical cancer, additional HPV PCR or mRNA in situ hybridization should be used to differentiate HPV-associated squamous cell carcinoma.

HPV analysis and immunostainings for p63 and S100 should be useful adjuncts to diagnose the 'basaloid' carcinoma of the uterine cervix. It may also be helpful to observe true ductal formation with microvilli by electron microscopy.

\section{Abbreviations}

BSCC: Basaloid squamous cell carcinoma; ACC: adenoid cystic carcinoma; HPV: Human Papilloma Virus

\section{Declarations}

\section{Acknowledgements}

I would like to thank Dr. Kyu-Rae Kim for diagnostic consultation.

\section{Author's contributions}

SAH: Writing-Reviewing and Editing, SHY: Conceptualization, Data curation, Visualization, Writing-Original draft preparation, Supervision

\section{Funding}


Not applicable.

\section{Ethics declarations}

This case study was approved by the Institutional Ethics Committee of Inje University Sanggye Paik Hospital, Seoul, Korea.

\section{Competing interests}

The author declares no conflict of interest.

\section{References}

1. Fritsch VA, Lentsch EJ: Basaloid squamous cell carcinoma of the head and neck: location means everything. Journal of surgical oncology 2014, 109(6):616-622.

2. Bellizzi AM, Woodford RL, Moskaluk CA, Jones DR, Kozower BD, Stelow EB: Basaloid squamous cell carcinoma of the esophagus: assessment for high-risk human papillomavirus and related molecular markers. The American journal of surgical pathology 2009, 33(11):1608-1614.

3. Chetty R, Serra S, Hsieh E: Basaloid squamous carcinoma of the anal canal with an adenoid cystic pattern: histologic and immunohistochemical reappraisal of an unusual variant. The American journal of surgical pathology 2005, 29(12):1668-1672.

4. Wang LC, Wang L, Kwauk S, Woo JA, Wu LQ, Zhu H, Zhan LZ, Sun NL, Zhang L: Analysis on the clinical features of 22 basaloid squamous cell carcinoma of the lung. Journal of cardiothoracic surgery 2011, 6:10.

5. Ginori A, Barone A, Santopietro R, Barbanti G, Cecconi F, Tripodi SA: Human papillomavirus-related basaloid squamous cell carcinoma of the bladder associated with genital tract human papillomavirus infection. International journal of urology : official journal of the Japanese Urological Association 2015, 22(2):222-225.

6. Daroca PJ, Jr., Dhurandhar HN: Basaloid carcinoma of uterine cervix. The American journal of surgical pathology 1980, 4(3):235-239.

7. Li TJ, Zhang YX, Wen J, Cowan DF, Hart J, Xiao SY: Basaloid squamous cell carcinoma of the esophagus with or without adenoid cystic features. Archives of pathology \& laboratory medicine 2004, 128(10):1124-1130.

8. Xing D, Bakhsh S, Melnyk N, Isacson C, Ho J, Huntsman DG, Gilks CB, Ronnett BM, Horlings HM: Frequent NFIB-associated Gene Rearrangement in Adenoid Cystic Carcinoma of the Vulva. International journal of gynecological pathology : official journal of the International Society of Gynecological Pathologists 2016.

9. Parwani AV, Smith Sehdev AE, Kurman RJ, Ronnett BM: Cervical adenoid basal tumors comprised of adenoid basal epithelioma associated with various types of invasive carcinoma: clinicopathologic 
features, human papillomavirus DNA detection, and P16 expression. Human pathology 2005, 36(1):82-90.

10. Moody CA, Laimins LA: Human papillomavirus oncoproteins: pathways to transformation. Nat Rev Cancer 2010, 10(8):550-560.

11. Boland JM, McPhail ED, Garcia JJ, Lewis JE, Schembri-Wismayer DJ: Detection of human papilloma virus and p16 expression in high-grade adenoid cystic carcinoma of the head and neck. Modern pathology : an official journal of the United States and Canadian Academy of Pathology, Inc 2012, 25(4):529-536.

12. Yang YJ, Gordon GB: Cervical adenoid cystic carcinoma coexisting with multiple human papillomavirus-associated genital lesions. A common etiology? Gynecologic and obstetric investigation 1999, 47(4):272-277.

13. Witkiewicz AK, Knudsen KE, Dicker AP, Knudsen ES: The meaning of p16(ink4a) expression in tumors: functional significance, clinical associations and future developments. Cell Cycle 2011, 10(15):2497-2503.

\section{Figures}




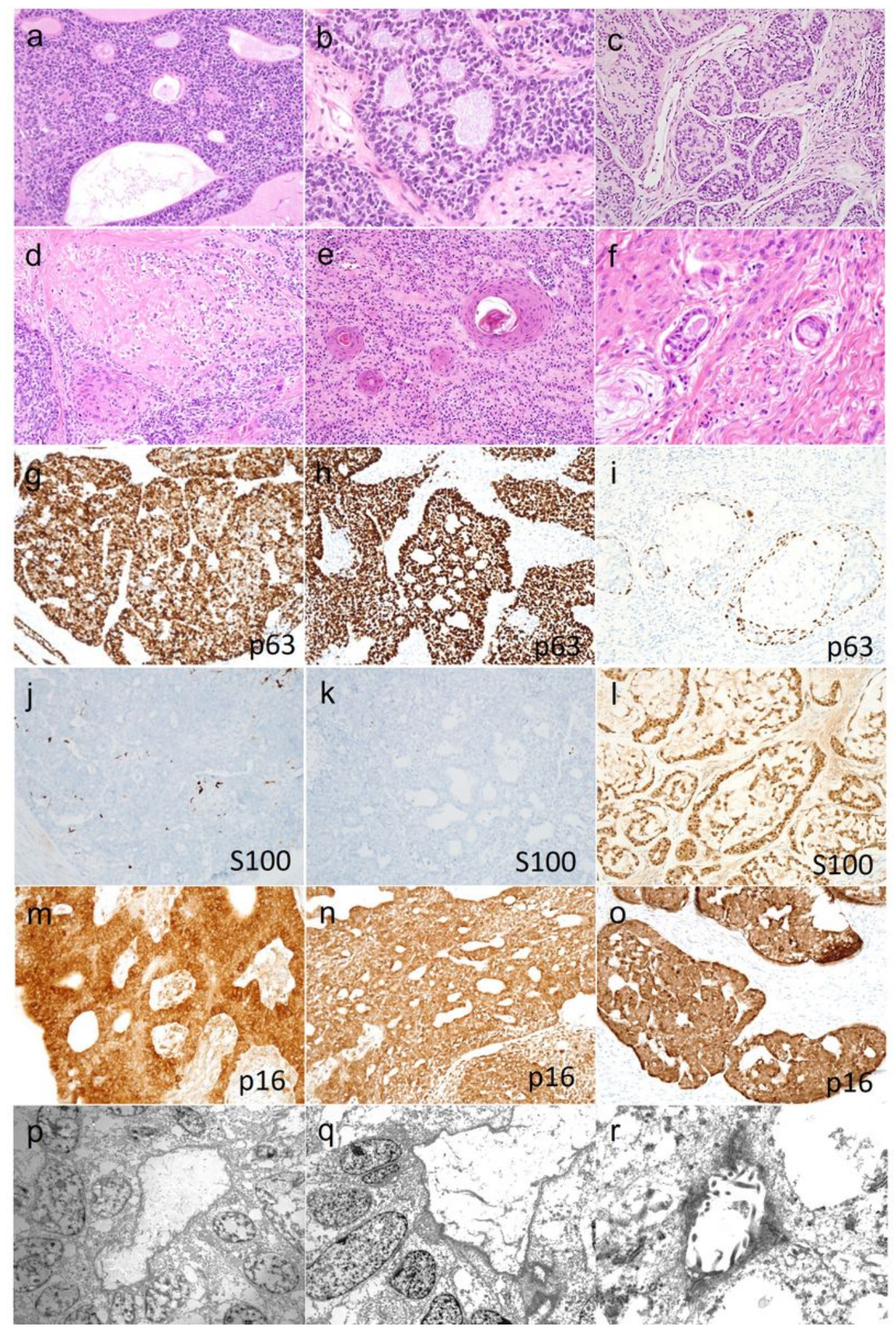

\section{Figure 1}

Despite similar cribriform and cystic features (A-C), two BSCCs have significantly higher cellular pleomorphism and distinct squamous differentiation (D\&E). ACC shows a glandular pattern with ductal differentiation at the periphery of the tumor (F, arrow). BSCCs are diffusely immunoreactive for p63 (G\&H) and p16 (M\&N), but immunonegative for $\mathrm{S} 100$ (J\&K). ACC shows selective peripheral/internalized positivity for p63 (I) and S100 (L) in myoepithelial cells, and diffuse expression for p16 (0) with no 
detection of HPV infection. Ultrastructural studies showed relatively undifferentiated cells consisting of a cystic lumen without their luminal microvilli (P\&Q); however, ACC shows occasional ductal differentiation with luminal microvilli ( $R$, arrow). 